\title{
ON THE SPATIAL ANALYTICITY OF SOLUTIONS TO THE KELLER-SEGEL EQUATIONS
}

\author{
OKIHIRO SAWADA \\ Department of Mathematical Science, Waseda University \\ Okubo 3-4-1, Shinjuku 169-8555, Tokyo, Japan \\ E-mail:sawada@gm.math.waseda.ac.jp
}

\begin{abstract}
The regularizing rate of solutions to the Keller-Segel equations in the whole space is estimated just as for the heat equation. As an application of these rate estimates, it is proved that the solution is analytic in spatial variables. Spatial analyticity implies that the propagation speed is infinite, i.e., the support of the solution coincides with the whole space for any short time, even if the support of the initial datum is compact.
\end{abstract}

1. Introduction and main results. Let us consider the following system of coupled reaction-diffusion equations in $\mathbf{R}^{n}$ for $n \in \mathbf{N}$ :

$$
\begin{cases}u_{t}=\Delta u-\nabla \cdot(u \nabla v) & \text { in } \mathbf{R}^{n} \times(0, T), \\ v_{t}=\Delta v-v+u & \text { in } \mathbf{R}^{n} \times(0, T), \\ \left.u\right|_{t=0}=u_{0},\left.\quad v\right|_{t=0}=v_{0} & \text { in } \mathbf{R}^{n} .\end{cases}
$$

In [10] E. F. Keller and L. A. Segel introduced (KS) as the mathematical model of the cell movement of mycetozoan by chemotaxis. Here $u:=u(t):=u(x, t)$ denotes the density of cells, and $v:=v(t):=v(x, t)$ stands for the concentration of chemoattractant at time $t \in(0, T)$ and location $x \in \mathbf{R}^{n}$; the initial data $u_{0}:=u_{0}(x)$ and $v_{0}:=v_{0}(x)$ are given non-negative functions. We use conventional notations: $u_{t}:=\partial u / \partial t, \Delta:=\sum_{i=1}^{n} \partial_{i}^{2}, \partial_{i}:=$ $\partial / \partial x_{i}, \nabla:=\left(\partial_{1}, \ldots, \partial_{n}\right), \nabla \cdot F:=\operatorname{div} F:=\sum_{i=1}^{n} \partial_{i} F^{i}$ for a vector $F=\left(F^{1}, \ldots, F^{n}\right)$.

There are a lot of works dealing with (KS). For example, local or global in time existence of smooth solutions in bounded domains with no-flux boundary conditions were obtained by [1]. Other related works can be found in references of [1].

2000 Mathematics Subject Classification: Primary 35Q30.

Key words and phrases: Keller-Segel equations, regularizing rate, spatial analyticity, propagation speed.

Research of the author partly supported by JSPS.

The paper is in final form and no version of it will be published elsewhere. 
Our aim is to derive the regularizing rate of solutions $(u, v)$ in short time, when $u_{0} \in L^{p}\left(\mathbf{R}^{n}\right)$ and $\nabla v_{0} \in\left(L^{p}\left(\mathbf{R}^{n}\right)\right)^{n}$. Throughout this note we often discuss $\nabla v$, instead of $v$ itself. Here $L^{p}\left(\mathbf{R}^{n}\right)$ is the Lebesgue space for $p \in[1, \infty]$ with the norm denoted by $\|\cdot\|_{p}$. We sometimes suppress the domain $\mathbf{R}^{n}$ in the notation, i.e., $L^{p}=L^{p}\left(\mathbf{R}^{n}\right)$. Also, we often do not distinguish the function spaces of scalar valued and vector valued functions, if no confusion is likely.

We state the main theorem in this note on the existence, uniqueness and analyticity in $x$ of solutions $(u, v)$ to $(\mathrm{KS})$ in $L^{p}$-framework:

Theorem 1.1. Let $n \in \mathbf{N}$ and $\max (1, n / 2)<p_{1} \leq p_{2}<\infty$ satisfying $1 / p_{1}+1 / p_{2} \leq 2 / n$, and let $p_{3} \in[1, \infty]$. Assume that $u_{0} \in L^{p_{1}}\left(\mathbf{R}^{n}\right), \nabla v_{0} \in\left(L^{p_{2}}\left(\mathbf{R}^{n}\right)\right)^{n}$, and that $v_{0} \in$ $L^{p_{3}}\left(\mathbf{R}^{n}\right)$. Then there exist $T_{0}>0$ and a unique solution $(u, v)$ in the class

$$
\left[t \mapsto t^{\frac{n}{2}\left(\frac{1}{p_{1}}-\frac{1}{q}\right)} u(t)\right] \in C\left(\left[0, T_{0}\right] ; L^{q}\left(\mathbf{R}^{n}\right)\right) \quad \text { for } q \in\left[p_{1}, \infty\right],
$$

and

$$
\left[t \mapsto t^{\frac{n}{2}\left(\frac{1}{p_{2}}-\frac{1}{q}\right)} \nabla v(t)\right] \in C\left(\left[0, T_{0}\right] ;\left(L^{q}\left(\mathbf{R}^{n}\right)\right)^{n}\right) \quad \text { for } q \in\left[p_{2}, \infty\right] .
$$

Moreover, $u, v \in C^{\infty}\left(\mathbf{R}^{n} \times\left(0, T_{0}\right)\right)$. Furthermore, $u$ and $v$ are analytic in $x$.

REMARK 1.2. (i) One can get mild solutions (the solution of the integral equations, see below) without the assumption $v_{0} \in L^{p_{3}}$. We, however, need to show that $(u, v)$ solves $(\mathrm{KS})$ in the classical sense. It is not necessary to impose non-negativity of $u_{0}$ and $v_{0}$ in Theorem 1.1.

(ii) For $u_{0}$ or $\nabla v_{0} \in L^{\infty}$ one can also get the similar results except the continuity in time at $t=0$, since the heat semigroup $e^{t \Delta}$ is not a $C_{0}$-semigroup in $L^{\infty}$. To obtain the continuity in time we restrict ourselves to $u_{0}$ or $\nabla v_{0}$ in $B U C\left(\mathbf{R}^{n}\right)$ or $\dot{B}_{\infty, 1}^{0}\left(\mathbf{R}^{n}\right)$, since $e^{t \Delta}$ is a bounded $C_{0}$-semigroup in these spaces, where $B U C$ is the space of all bounded and uniformly continuous functions, and $\dot{B}_{\infty, 1}^{0}$ is the homogeneous Besov space; see the details in $[2,5,13]$.

(iii) Propagation speed is infinite. As the corollary of analyticity in $x$, it is shown that the propagation speed of solutions to $(\mathrm{KS})$ is infinite. That is to say, the supports of $u(t)$ and $v(t)$ coincide with $\mathbf{R}^{n}$ for any small $t>0$, even if the supports of $u_{0}$ and $v_{0}$ are compact.

(iv) Other equations. We may obtain the same results (in particular, analyticity in $x$ ) on the Cauchy problem of the following three equations of parabolic type.

(a) The generalized Keller-Segel equations:

$$
\left\{\begin{aligned}
u_{t} & =\Delta u-\nabla \cdot\left(u^{q} \nabla v\right), \\
\tau v_{t} & =\Delta v-\gamma v+u
\end{aligned}\right.
$$

for $q \in \mathbf{N}, \gamma \in \mathbf{R}$ and $\tau=0,1$ with initial data $u_{0} \in L^{p}$ for $p \geq n$ if $\tau=0$, and $u_{0} \in L^{p_{1}}$, $\nabla v_{0} \in\left(L^{p_{2}}\right)^{n}$ if $\tau=1$. For the case when $\tau=0$ and $q=1$, (GKS) is the so-called Nagai model, there exists a blow-up solution in finite time, see [11]. When $\gamma=0$ and $q=1$, one can obtain the global in time smooth solution for sufficiently small data, which was shown by H. Kozono and Y. Sugiyama, recently. 
(b) The Fujita equation (semilinear heat equation) with algebraic nonlinearity:

$$
u_{t}=\Delta u+|u|^{q-1} u
$$

for $q \in \mathbf{N}$ with $u_{0} \in B U C$; it is not necessary to impose any positivity.

(c) The Allen-Cahn equation:

$$
u_{t}=\varepsilon \Delta u-u^{3}+u
$$

for $\varepsilon>0$ with suitable initial data, e.g. $u_{0} \in B U C\left(\mathbf{R}^{n}\right)$ and $-1 \leq u_{0} \leq 1$.

In the proof of Theorem 1.1 we use integral equations, which will be explained later. Existence is based on an iteration scheme, see e.g. [9], that is, we use successive approximations. Uniqueness follows from the Gronwall inequality. Involving the higher order derivatives, the iteration procedure works again to show that $u$ and $v$ are smooth. To get the analyticity of $u$ and $v$ in $x$ we establish the rate estimate for the higher order derivatives.

Let us recall the heat equation:

$$
w_{t}-\Delta w=0,\left.\quad w\right|_{t=0}=f \in L^{r}\left(\mathbf{R}^{n}\right) .
$$

Using the heat semigroup $e^{t \Delta}:=G_{t} *$ and $G_{t}(x):=(4 \pi t)^{-\frac{n}{2}} \exp \left(-\frac{|x|^{2}}{4 t}\right)$, the solution $w$ is given in the form $w=e^{t \Delta} f$ enjoying the following regularizing rate estimates:

$$
\left\|\partial_{x}^{\beta} w(t)\right\|_{q} \leq C_{0}|\beta|^{\frac{|\beta|}{2}} t^{-\frac{|\beta|}{2}-\frac{n}{2}\left(\frac{1}{r}-\frac{1}{q}\right)}\|f\|_{r} \quad \text { for } t>0 \text { and } 1 \leq r \leq q \leq \infty
$$

with some constant $C_{0}$ depending only on $n$. Here $\partial_{x}^{\beta}:=\partial_{1}^{\beta_{1}} \cdots \partial_{n}^{\beta_{n}}$ and $|\beta|:=\beta_{1}+\cdots+\beta_{n}$ for multi-index $\beta=\left(\beta_{1}, \ldots, \beta_{n}\right) \in \mathbf{N}_{0}^{n}$, where $\mathbf{N}_{0}=\mathbf{N} \cup\{0\}$. Obviously, (3) implies that $w(t)$ is analytic in $x$ for all $t>0$.

We are now in a position to introduce the notion of a mild solution. Historically, this notion was introduced by F. E. Browder to study equations of parabolic type, and by H. Fujita and T. Kato [3] for the nonstationary Navier-Stokes equations. By Duhamel's principle it is straightforward to solve $(\mathrm{KS})$ in time to derive the integral equations:

$$
\left\{\begin{aligned}
u(t) & =e^{t \Delta} u_{0}-\int_{0}^{t} e^{(t-s) \Delta} \nabla \cdot(u(s) \nabla v(s)) d s, \\
\nabla v(t) & =e^{t \Delta} \nabla v_{0}-\int_{0}^{t} \nabla e^{(t-s) \Delta}(u(s)-v(s)) d s .
\end{aligned}\right.
$$

Obviously, (INT) is equivalent to $(\mathrm{KS})$. The pair of solutions $(u, v)$ to (INT) is often called a mild solution, we also use this terminology. In what follows we mainly deal with (INT) rather than (KS).

In this note we derive estimates of higher order derivatives of $u$ and $v$ similar to (3): Proposition 1.3. Let $n \in \mathbf{N}, p>1, p \in[n, \infty)$, and let $u_{0}, \nabla v_{0} \in L^{p}\left(\mathbf{R}^{n}\right)$. Assume that $(u(t), v(t))$ is a mild solution on $t \in(0, T)$ such that $u, \nabla v \in C^{\infty}\left(\mathbf{R}^{n} \times(0, T)\right)$ and

$$
u, \nabla v \in C\left([0, T) ; L^{p}\left(\mathbf{R}^{n}\right)\right) \cap C\left((0, T) ; L^{r}\left(\mathbf{R}^{n}\right)\right)
$$

for some $T \in(0,1]$ and some $r>\max (p, 2)$. Let $M_{j}$ for $j=1, \ldots, 4$ be constants satisfying

$$
\begin{array}{ll}
M_{1} \geq \sup _{0 \leq t<T}\|u(t)\|_{p}, & M_{2} \geq \sup _{0<t<T} t^{\frac{n}{2}\left(\frac{1}{p}-\frac{1}{r}\right)}\|u(t)\|_{r}, \\
M_{3} \geq \sup _{0 \leq t<T}\|\nabla v(t)\|_{p}, & M_{4} \geq \sup _{0<t<T} t^{\frac{n}{2}\left(\frac{1}{p}-\frac{1}{r}\right)}\|\nabla v(t)\|_{r} .
\end{array}
$$


Then there exist positive constants $D_{1}$ and $D_{2}$ depending only on $n, p, r$ and $M_{j}$ such that

$$
\left\|\partial_{x}^{\beta} u(t)\right\|_{q}+\left\|\partial_{x}^{\beta} \nabla v(t)\right\|_{q} \leq D_{1}\left(D_{2}|\beta|\right)^{|\beta|} t^{-\frac{|\beta|}{2}-\frac{n}{2}\left(\frac{1}{p}-\frac{1}{q}\right)}
$$

for all $q \in[p, \infty], t \in(0, T)$ and $\beta \in \mathbf{N}_{0}^{n}$.

For simplicity we only deal with the solution when $p_{1}=p_{2}$ in Theorem 1.1. From (4) it is easy to see that $u$ and $v$ are analytic in $x$. Indeed, one can estimate the radius of convergence of Taylor's expansion of $u(=: \rho(t))$ from below:

$$
\rho(t)=\left[\limsup _{k \rightarrow \infty}\left(\frac{\left\|\partial_{i}^{k} u(t)\right\|_{\infty}}{k !}\right)^{1 / k}\right]^{-1} \geq C \sqrt{t}
$$

for some constant $C$ for any $t \in(0, T)$ and $i=1, \ldots, n$. The estimate (5) follows from Cauchy-Hadamard's criterion and Stirling's formula, easily. It can be also shown that $v(t) \in C^{\omega}\left(\mathbf{R}^{n}\right)$.

To prove Proposition 1.3 we use the technique developed by Y. Giga and the author of this note [6]; see also [12]. We divide the time interval $(0, t)$ of integrals in (INT) into two parts, to distribute the singularity. To integrate them we press every differentiation against the heat kernel for $s \in(0,(1-\varepsilon) t)$, and against $u$ and $v$ for $s \in((1-\varepsilon) t, t)$. Finally, we use the Gronwall type inequality (Lemma 2.3) to get (4). Also, smoothness of mild solutions can be shown by this technique.

This paper is organized as follows. In section 2 we recall several lemmata. Section 3 will be devoted to the proof of Theorem 1.1. In Proposition 3.1 we shall give a proof of existence and uniqueness of mild solutions. In Proposition 3.2 we shall verify that the mild solution is in the class $C^{\omega}$ in $x$.

Acknowledgments. The author would like to express his hearty gratitude to Professor Yoshie Sugiyama for her encouragement and valuable suggestions. The author would also like to thank Professor Takeshi Ohtsuka for his comments and suggestions on the Allen-Cahn equation and other equations. The author also wishes to express his thanks to the referee for her/his helpful pointing out mistakes in the first version of this paper, and for letting him know the article [1]. The work of the author was partly supported by the Japan Society for Promotion of Science.

2. Preliminaries. In this section we give some lemmata. Firstly, we verify (4).

Lemma 2.1. Let $n \in \mathbf{N}$. Then there exists a constant $C_{0}>0$ depending only on $n$ such that (3) holds for all $t>0,1 \leq r \leq q \leq \infty, \beta \in \mathbf{N}_{0}^{n}$ and $f \in L^{r}\left(\mathbf{R}^{n}\right)$.

Proof. Although this lemma was proved by [6, Lemma 2.1], we give its proof for the readers' convenience. Since $\left\|\partial_{i} G_{t}\right\|_{1} \leq \pi^{-1 / 2} t^{-1 / 2}$, by Young's inequality we have

$$
\left\|\partial_{i} e^{t \Delta} f\right\|_{r} \leq \pi^{-\frac{1}{2}} t^{-\frac{1}{2}}\|f\|_{r}, \quad\left\|e^{t \Delta} f\right\|_{q} \leq(4 \pi t)^{-\frac{n}{2}\left(\frac{1}{r}-\frac{1}{q}\right)}\|f\|_{r}
$$

for all $t>0,1 \leq r \leq \infty, i=1, \ldots, n$ and $f \in L^{r}\left(\mathbf{R}^{n}\right)$. Notice that the heat semigroup and spatial differentiation commute. Using the semigroup property, for $1<\theta, \theta^{\prime}<\infty$ 
satisfying $\frac{1}{\theta}+\frac{1}{\theta^{\prime}}=1$ we have

$$
\begin{aligned}
\left\|\partial_{x}^{\beta} e^{t \Delta} f\right\|_{q} & =\left\|e^{\frac{t}{\theta^{\prime}} \Delta} \prod_{i=1}^{n}\left(\partial_{i} e^{\frac{t}{\theta|\beta|} \Delta}\right)^{\beta_{i}} f\right\|_{q} \\
& \leq\left\|e^{\frac{t}{\theta^{\prime}} \Delta}\right\|_{\mathcal{L}\left(L^{r} \rightarrow L^{q}\right)}\left\{\prod_{i=1}^{n}\left\|\partial_{i} e^{\frac{t}{\theta|\beta|} \Delta}\right\|_{\mathcal{L}\left(L^{r} \rightarrow L^{r}\right)}^{\beta_{i}}\right\}\|f\|_{r} \\
& \leq\left(4 \pi \frac{t}{\theta^{\prime}}\right)^{-\frac{n}{2}\left(\frac{1}{r}-\frac{1}{q}\right)}\left\{\pi^{-\frac{1}{2}}\left(\frac{t}{\theta|\beta|}\right)^{-\frac{1}{2}}\right\}^{|\beta|}\|f\|_{r}
\end{aligned}
$$

for all $t>0,1 \leq r \leq q \leq \infty$ and $f \in L^{r}$. Finally, we take $\theta=\pi$ so that the constant $C_{0}:=(4 \pi-4)^{-n / 2}$ does not depend on $\beta$.

Next, we recall an estimate for multiplication of multi-sequences with binomial coefficient, which has been proved by C. Kahane [8, Lemma 2.1]. That will be used to compute the nonlinear term.

Lemma 2.2. Let $\delta>1 / 2$, and let $n \in \mathbf{N}$. Then there exists a positive constant $\lambda$ depending only on $\delta$ and $n$ such that

$$
\sum_{\gamma \leq \beta}\left(\begin{array}{l}
\beta \\
\gamma
\end{array}\right)|\gamma|^{|\gamma|-\delta}|\beta-\gamma|^{|\beta-\gamma|-\delta} \leq \lambda|\beta|^{|\beta|-\delta}
$$

for all $\beta \in \mathbf{N}_{0}^{n}$. Here $\gamma \leq \beta$ means $\gamma_{i} \leq \beta_{i}$ for all $i=1, \ldots, n$ for multi-indecies $\beta=\left(\beta_{1}, \ldots, \beta_{n}\right)$ and $\gamma=\left(\gamma_{1}, \ldots, \gamma_{n}\right)$, and $\left(\begin{array}{c}\beta \\ \gamma\end{array}\right):=\prod_{i=1}^{n} \frac{\beta_{i} !}{\gamma_{i} !\left(\beta_{i}-\gamma_{i}\right) !}$.

The dependence of $\lambda$ on $\delta$ is essentially $\lambda \sim \sum_{j=1}^{\infty} j^{-1 / 2-\delta}$, so we need $\delta>1 / 2$. The proof of Lemma 2.2 is based on Stirling's formula. We omit the proof for brevity.

At the end of this section, we refer to a Gronwall type inequality. Originally, the following lemma has been proved by M.-H. Giga and Y. Giga [4], and its modification (sequence version) is in [6].

Lemma 2.3. Let $T>0, \alpha \in \mathbf{R}$, and let $\mu \in(0,1)$. Assume that $\psi_{0}$ is non-negative, measurable and locally integrable in $(0, T)$, and that $\left\{\psi_{j}\right\}_{j=1}^{\infty}$ be a sequence of non-negative measurable functions in $(0, T)$. Assume that $t^{-\alpha} \psi_{0}(t)$ is bounded in $(0, T)$. Let $b_{\varepsilon}$ be nonincreasing with respect to $\varepsilon$. Assume that there is a positive constant $\sigma$ such that

$$
\psi_{0}(t) \leq b_{\varepsilon} t^{\alpha}+\sigma \int_{(1-\varepsilon) t}^{t}(t-s)^{-\mu} s^{-1+\mu} \psi_{0}(s) d s
$$

and

$$
\psi_{j+1}(t) \leq b_{\varepsilon} t^{\alpha}+\sigma \int_{(1-\varepsilon) t}^{t}(t-s)^{-\mu} s^{-1+\mu} \psi_{j}(s) d s
$$

for all $j \in \mathbf{N}_{0}, t \in(0, T)$ and $\varepsilon \in(0,1)$. Let $\varepsilon_{0}$ be a unique positive number such that $I\left(2 \varepsilon_{0}\right)=\min \left\{\frac{1}{2 \sigma}, I(1)\right\}$ with $I(\varepsilon):=\int_{1-\varepsilon}^{1}(1-\tau)^{-\mu} \tau^{\alpha-1+\mu} d \tau$. Then

$$
\psi_{j}(t) \leq 2 b_{\varepsilon_{0}} t^{\alpha}
$$

for all $j \in \mathbf{N}_{0}$ and $t \in(0, T)$.

We skip the proof, given in [6]. 
3. Proof of Theorem 1.1. We split the proof into three parts: (i) existence and uniqueness (by Proposition 3.1), (ii) smoothness, and (iii) analyticity (by Proposition 3.2).

Firstly, the time-local existence and uniqueness of mild solutions in $L^{n}$-framework. Although the proof of existence is based on the semigroup approach that is standard and explained in several books (e.g. [7]), for completeness we shall give the full proof. Throughout this section we only discuss the case $p_{1}=p_{2}=n$, since the proof with other exponents is essentially the same (or easier).

Proposition 3.1. Let $n \in \mathbf{N}, u_{0} \in L^{n}\left(\mathbf{R}^{n}\right)$, and let $\nabla v_{0} \in\left(L^{n}\left(\mathbf{R}^{n}\right)\right)^{n}$. Then there exist $T_{0}>0$ and a unique solution $(u, v)$ in the class $(1)-(2)$ for all $q \in[n, \infty]$.

Proof. Firstly, we construct mild solutions by an iteration scheme. We define the successive approximations starting at

$$
u_{1}(t):=e^{t \Delta} u_{0}, \quad \nabla v_{1}(t):=e^{t \Delta} \nabla v_{0},
$$

and define $\left\{u_{j}\right\}_{j}$ and $\left\{\nabla v_{j}\right\}_{j}$ by

$$
u_{j+1}(t):=u_{1}(t)-\int_{0}^{t} e^{(t-s) \Delta} \nabla \cdot\left(u_{j}(s) \nabla v_{j}(s)\right) d s
$$

and

$$
\nabla v_{j+1}(t):=\nabla v_{1}(t)-\int_{0}^{t} \nabla e^{(t-s) \Delta}\left(v_{j}(s)-u_{j}(s)\right) d s
$$

for all $j \in \mathbf{N}$. We shall verify that the pair $\left\{\left(u_{j}, \nabla v_{j}\right)\right\}_{j}$ is a Cauchy sequence in the class (1)-(2), and has a unique limit $(u, \nabla v)$ which satisfies (INT).

Let $\delta \in(0,1)$. Put

$$
K_{j}:=K_{j}(T):=\sup _{0<t<T} t^{\frac{1-\delta}{2}}\left\|u_{j}(t)\right\|_{\frac{n}{\delta}}, \quad L_{j}:=L_{j}(T):=\sup _{0<t<T} t^{\frac{1-\delta}{2}}\left\|\nabla v_{j}(t)\right\|_{\frac{n}{\delta}} .
$$

Remark that

$$
K_{1} \rightarrow 0, \quad L_{1} \rightarrow 0 \quad \text { as } T \rightarrow 0,
$$

since $C_{0}^{\infty}$ is a dense subset of $L^{p}$ for $p \in[1, \infty)$.

We shall derive a priori estimate for $u_{j}$. Taking $\|\cdot\|_{\frac{n}{\delta}}$ into (6), and multiplying $t^{\frac{1-\delta}{2}}$, we have

$$
\begin{aligned}
t^{\frac{1-\delta}{2}}\left\|u_{j+1}(t)\right\|_{\frac{n}{\delta}} & \leq K_{1}+t^{\frac{1-\delta}{2}} \int_{0}^{t}\left\|e^{(t-s) \Delta} \nabla \cdot\left(u_{j}(s) \nabla v_{j}(s)\right)\right\|_{\frac{n}{\delta}} d s \\
& \leq K_{1}+t^{\frac{1-\delta}{2}} \int_{0}^{t}\left\|e^{(t-s) \Delta} \nabla \cdot\right\|_{\mathcal{L}\left(L^{\frac{n}{2 \delta}} \rightarrow L^{\frac{n}{\delta}}\right)}\left\|u_{j}(s) \nabla v_{j}(s)\right\|_{\frac{n}{2 \delta}} d s \\
& \leq K_{1}+C t^{\frac{1-\delta}{2}} \int_{0}^{t}(t-s)^{-\frac{n}{2}\left(\frac{2 \delta}{n}-\frac{\delta}{n}\right)-\frac{1}{2}}\left\|u_{j}(s)\right\|_{\frac{n}{\delta}}\left\|\nabla v_{j}(s)\right\|_{\frac{n}{\delta}} d s \\
& \leq K_{1}+C t^{\frac{1-\delta}{2}} K_{j} L_{j} \int_{0}^{t}(t-s)^{-\frac{n}{2}\left(\frac{2 \delta}{n}-\frac{\delta}{n}\right)-\frac{1}{2}} s^{-1+\delta} d s \\
& \leq K_{1}+C_{1} K_{j} L_{j},
\end{aligned}
$$

where the constant $C_{1}$ depends only on $n$ and $\delta$. Here we have used Hölder's inequality and Lemma 2.1. Taking $\sup _{0<t<T}$ on both sides, we thus have

$$
K_{j+1} \leq K_{1}+C_{1} K_{j} L_{j}
$$


Similarly, for (7) we derive

$$
\begin{aligned}
t^{\frac{1-\delta}{2}}\left\|\nabla v_{j+1}(t)\right\|_{\frac{n}{\delta}} & \leq L_{1}+t^{\frac{1-\delta}{2}} \int_{0}^{t}\left\|\nabla e^{(t-s) \Delta}\left(v_{j}(s)-u_{j}(s)\right)\right\|_{\frac{n}{\delta}} d s \\
& \leq L_{1}+C t^{\frac{1-\delta}{2}} \int_{0}^{t}(t-s)^{-\frac{1}{2}}\left\|u_{j}(s)\right\|_{\frac{n}{\delta}} d s+C t^{\frac{1-\delta}{2}} \int_{0}^{t}\left\|\nabla v_{j}(s)\right\|_{\frac{n}{\delta}} d s \\
& \leq L_{1}+C t^{\frac{1-\delta}{2}} K_{j} \int_{0}^{t}(t-s)^{-\frac{1}{2}} s^{-\frac{1-\delta}{2}} d s+C t^{\frac{1-\delta}{2}} L_{j} \int_{0}^{t} s^{-\frac{1-\delta}{2}} d s \\
& \leq L_{1}+C_{2} \sqrt{t} K_{j}+C_{3} t L_{j}
\end{aligned}
$$

with some positive constants $C_{2}$ and $C_{3}$ which depend only on $n$ and $\delta$. So, we take $\sup _{0<t<T}$ to get

$$
L_{j+1} \leq L_{1}+C_{2} \sqrt{T} K_{j}+C_{3} T L_{j}
$$

Notice that $\left\{u_{j}\right\}$ and $\left\{\nabla v_{j}\right\}$ are uniformly bounded with values in $L^{\frac{n}{\delta}}$ on $\left(0, T_{0}\right)$ for some small $T_{0}$ from (9) and (10). Indeed, by (8) for any $\lambda>0$ there exists $T_{\lambda}$ such that $K_{1} \leq \lambda$ and $L_{1} \leq \lambda$ for all $T \leq T_{\lambda}$. Set $\lambda=\lambda_{*}:=\frac{1}{6 C_{1}}$ and $T_{0}:=\min \left(T_{\lambda_{*}}, \frac{1}{4 C_{2}^{2}}, \frac{1}{3 C_{3}}\right)$. We therefore obtain

$$
\sup _{j \in \mathbf{N}} K_{j}(T) \leq 2 \lambda \quad \text { and } \quad \sup _{j \in \mathbf{N}} L_{j}(T) \leq 3 \lambda \quad \text { for } \quad T \leq T_{0} .
$$

Using uniform boundedness (11), one can show that $u_{j}$ belongs to the class (1) as well as $\nabla v_{j}$ belongs to (2) for all $j \in \mathbf{N}$ and for all $q \in[n, \infty]$ at least when $T \leq T_{0}$. One can also easily see that $\left\{t^{\frac{n}{2}\left(\frac{1}{n}-\frac{1}{q}\right)} u_{j}(t)\right\}$ and $\left\{t^{\frac{n}{2}\left(\frac{1}{n}-\frac{1}{q}\right)} \nabla v_{j}(t)\right\}$ are continuous in time with values in $L^{q}$ for all $q \in[n, \infty]$. Moreover, they are Cauchy sequences in $C\left(\left[0, T_{0}\right] ; L^{q}\right)$ if we again choose $T_{0}$ small enough. So, there exists limit $(u, \nabla v)$ satisfying (INT). Uniqueness follows from Gronwall's inequality, see e.g. [5]. This completes the proof of Proposition 3.1 .

Smoothness of a mild solution $(u, v)$ is also obtained by a modification of the proof above. In order to get the $\ell$-th derivative in $x$, we involve the quantities

$$
K_{j}^{(\ell)}:=\sup _{0<t<T} t^{\frac{1-\delta}{2}+\frac{\ell}{2}}\left\|\nabla^{\ell} u_{j}(t)\right\|_{\frac{n}{\delta}}, \quad L_{j}^{(\ell)}:=\sup _{0<t<T} t^{\frac{1-\delta}{2}+\frac{\ell}{2}}\left\|\nabla^{\ell+1} v_{j}(t)\right\|_{\frac{n}{\delta}}
$$

for $\ell \in \mathbf{N}$ in the iteration scheme. We use induction with respect to $\ell$, and divide the time-interval of integrals into $(0, t / 2)$ and $(t / 2, t)$. For example, we derive the estimate of the second derivative of $u_{j}$ by

$$
\begin{aligned}
& t^{\frac{3-\delta}{2}}\left\|\nabla^{2} u_{j+1}(t)\right\|_{\frac{n}{\delta}} \\
& \quad \leq t^{\frac{3-\delta}{2}}\left\|\nabla^{2} e^{t \Delta} u_{0}\right\|_{\frac{n}{\delta}}+t^{\frac{3-\delta}{2}}\left(\int_{0}^{t / 2}+\int_{t / 2}^{t}\right)\left\|\nabla^{2} e^{(t-s) \Delta} \nabla \cdot\left(u_{j}(s) \nabla v_{j}(s)\right)\right\|_{\frac{n}{\delta}} d s \\
& \quad \leq K_{1}^{(2)}+C\left(K_{j}^{(1)} L_{j}+K_{j} L_{j}^{(1)}+K_{j}^{(2)} L_{j}+K_{j}^{(1)} L_{j}^{(1)}+K_{j} L_{j}^{(2)}\right) .
\end{aligned}
$$

It is natural that we have to choose $T_{\ell}$ small enough so that $K_{j}^{(\ell)}$ and $L_{j}^{(\ell)}$ are uniformly bounded in this way. Indeed, we may see $T_{\ell} \sim \ell^{-\ell}$ basically, if we use above arguments. 
However, it is not necessary to choose $T_{\ell}$ depending on $\ell$ for deriving uniform bounds of $K_{j}^{(\ell)}$ and $L_{j}^{(\ell)}$ on $j$ actually, if we use a modification of the proof of the next proposition; see the end of this section.

We may extend the time-interval when the mild solution exists up to $T_{0}$, since mild solution does not blow-up and is unique at least until $T_{0}$. So, it is shown that our mild solution $(u(t), v(t))$ belongs to $C^{\infty}\left(\mathbf{R}^{n}\right)$ for all $t \in\left(0, T_{0}\right)$. The analyticity of the heat semigroup implies that $u, v \in C^{\infty}\left(\mathbf{R}^{n} \times\left(0, T_{0}\right)\right)$.

Next we establish the estimates for higher order derivatives of $u$ and $\nabla v$, which are formally equivalent to (4). Again, we only discuss the case $p=n$ in what follows.

Proposition 3.2. Suppose that all assumptions of Proposition 1.3 hold with $p=n$ and $n \geq 2$. Let $\delta \in(1 / 2,1]$. Then there exist positive constants $D_{1}$ and $D_{2}$ depending only on $n, r, \delta$ and $M_{j}$ such that

$$
\left\|\partial_{x}^{\beta} u(t)\right\|_{q}+\left\|\partial_{x}^{\beta} \nabla v(t)\right\|_{q} \leq D_{1}\left(D_{2}|\beta|\right)^{|\beta|-\delta} t^{-\frac{|\beta|}{2}-\frac{n}{2}\left(\frac{1}{n}-\frac{1}{q}\right)}
$$

for all $q \in[n, \infty], t \in(0, T)$ and $\beta \in \mathbf{N}_{0}^{n}$.

Proof. Let $n \geq 2, r>n$ and $\delta \in(1 / 2,1]$. Assume that $u_{0}, \nabla v_{0} \in L^{n}\left(\mathbf{R}^{n}\right)$. Let $T \leq$ $\min \left(1, T_{0}\right)$, where $\left(0, T_{0}\right)$ is the time-interval when the unique solution exists ensured by Theorem 1.1. Using the above arguments, for $m_{0} \in \mathbf{N}$ (fixed later) we choose $D_{1}$ large enough such that

$$
\left\|\partial_{x}^{\beta} u(t)\right\|_{q}+\left\|\partial_{x}^{\beta} \nabla v(t)\right\|_{q} \leq D_{1}, \quad t \in\left(0, T_{0}\right)
$$

hold for all $\beta \in \mathbf{N}_{0}^{n}$ with $|\beta| \leq m_{0}$. Clearly, (13) implies (12) for $|\beta| \leq m_{0}$. We shall derive (12) by induction. Let $m \in \mathbf{N}$ and $m \geq m_{0}$. Assume that (12) holds for all $|\beta| \leq m-1$. We now proceed to show (12) when $|\beta|=m$.

Let $\varepsilon \in(0,1 / 2)$. By (INT) we estimate the $L^{q}$-norm of $\partial_{x}^{\beta} u$ :

$$
\begin{aligned}
\left\|\partial_{x}^{\beta} u(t)\right\|_{q} & \leq\left\|\partial_{x}^{\beta} e^{t \Delta} u_{0}\right\|_{q}+\left(\int_{0}^{(1-\varepsilon) t}+\int_{(1-\varepsilon) t}^{t}\right)\left\|\partial_{x}^{\beta} e^{(t-s) \Delta} \nabla \cdot(u(s) \nabla v(s))\right\|_{q} d s \\
& =: U_{1}+U_{2}+U_{3} .
\end{aligned}
$$

We derive the estimates for each term. For $U_{1}$ by Lemma 2.1 we have

$$
U_{1} \leq \tilde{C}_{1}|\beta|^{\frac{|\beta|}{2}} t^{-\frac{|\beta|}{2}-\frac{n}{2}\left(\frac{1}{n}-\frac{1}{q}\right)} .
$$

Here the constant $\tilde{C}_{1}:=C_{0}\left\|u_{0}\right\|_{n} \leq C_{0} M_{1}$ does not depend on $\beta$ and $t$. We estimate $U_{2}$ by

$$
\begin{aligned}
U_{2} & \leq \int_{0}^{(1-\varepsilon) t}\left\|\partial_{x}^{\beta} e^{(t-s) \Delta} \nabla \cdot\right\|_{\mathcal{L}\left(L^{\frac{n}{2}} \rightarrow L^{q}\right)}\|u(s) \nabla v(s)\|_{\frac{n}{2}} d s \\
& \leq C_{0}(|\beta|+1)^{\frac{|\beta|+1}{2}} \int_{0}^{(1-\varepsilon) t}(t-s)^{-\frac{|\beta|+1}{2}-\frac{n}{2}\left(\frac{2}{n}-\frac{1}{q}\right)}\|u(s)\|_{n}\|\nabla v(s)\|_{n} d s \\
& \leq \tilde{C}_{2}(|\beta|+1)^{\frac{|\beta|+1}{2}} \varepsilon^{-\frac{|\beta|}{2}-\frac{3}{2}+\frac{n}{2 q}} t^{-\frac{|\beta|}{2}-\frac{1}{2}+\frac{n}{2 q}} .
\end{aligned}
$$

Here $\tilde{C}_{2}:=2 C_{0} M_{1} M_{3}$. We divide $U_{3}$ into two parts: 


$$
\begin{aligned}
U_{3}= & \int_{(1-\varepsilon) t}^{t}\left\|e^{(t-s) \Delta} \nabla \cdot \partial_{x}^{\beta}(u(s) \nabla v(s))\right\|_{q} d s \\
\leq & \int_{(1-\varepsilon) t}^{t}\left\|e^{(t-s) \Delta} \nabla \cdot\right\|_{\mathcal{L}\left(L^{\frac{q r}{q+r}} \rightarrow L^{q}\right)}\left\|\sum_{\gamma \leq \beta}\left(\begin{array}{c}
\beta \\
\gamma
\end{array}\right)\left(\partial_{x}^{\gamma} u\right)\left(\partial_{x}^{\beta-\gamma} \nabla v\right)\right\|_{\frac{q r}{q+r}} d s \\
\leq & C_{0} \int_{(1-\varepsilon) t}^{t}(t-s)^{-\frac{1}{2}-\frac{1}{r}}\left(\left\|\partial_{x}^{\beta} u\right\|_{q}\|\nabla v\|_{r}+\|u\|_{r}\left\|\partial_{x}^{\beta} \nabla v\right\|_{q}\right) d s \\
& +C_{0} \int_{(1-\varepsilon) t}^{t}(t-s)^{-\frac{1}{2}-\frac{1}{r}} \sum_{0<\gamma<\beta}\left(\begin{array}{c}
\beta \\
\gamma
\end{array}\right)\left\|\partial_{x}^{\gamma} u\right\|_{q}\left\|\partial_{x}^{\beta-\gamma} \nabla v\right\|_{r} d s \\
= & : U_{3 a}+U_{3 b .}
\end{aligned}
$$

Here we used that $\gamma<\beta$ means $\gamma \leq \beta$ and $\gamma_{i}<\beta_{i}$ for some $i$. By the definition of $M_{2}$ and $M_{4}$ we easily estimate $U_{3 a}$ by

$$
U_{3 a} \leq \tilde{C}_{3} \int_{(1-\varepsilon) t}^{t}(t-s)^{-\frac{1}{2}-\frac{1}{r}} s^{-\frac{1}{2}+\frac{1}{r}}\left(\left\|\partial_{x}^{\beta} u(s)\right\|_{q}+\left\|\partial_{x}^{\beta} \nabla v(s)\right\|_{q}\right) d s
$$

with $\tilde{C}_{3}:=C_{0} \max \left(M_{2}, M_{4}\right)$. For $U_{3 b}$ we use the assumptions of induction:

$$
\begin{aligned}
U_{3 b} \leq & C_{0} \int_{(1-\varepsilon) t}^{t}(t-s)^{-\frac{1}{2}-\frac{1}{r}} \sum_{0<\gamma<\beta}\left(\begin{array}{c}
\beta \\
\gamma
\end{array}\right) D_{1}\left(D_{2}|\gamma|\right)^{|\gamma|-\delta} s^{-\frac{|\gamma|}{2}-\frac{n}{2}\left(\frac{1}{n}-\frac{1}{q}\right)} \\
& \times D_{1}\left(D_{2}|\beta-\gamma|\right)^{|\beta-\gamma|-\delta} s^{-\frac{|\beta-\gamma|}{2}-\frac{n}{2}\left(\frac{1}{n}-\frac{1}{r}\right)} d s \\
= & C_{0} D_{1}^{2} D_{2}^{|\beta|-2 \delta} J_{\varepsilon,|\beta|} t^{-\frac{|\beta|}{2}-\frac{1}{2}+\frac{n}{2 q}} \sum_{0<\gamma<\beta}\left(\begin{array}{c}
\beta \\
\gamma
\end{array}\right)|\gamma|^{|\gamma|-\delta}|\beta-\gamma|^{|\beta-\gamma|-\delta} \\
\leq & C_{0} \lambda J_{\varepsilon,|\beta|} D_{1}^{2} D_{2}^{|\beta|-2 \delta}|\beta|^{|\beta|-\delta} t^{-\frac{|\beta|}{2}-\frac{1}{2}+\frac{n}{2 q}} .
\end{aligned}
$$

Here we have used Lemma 2.2 , and $J_{\varepsilon,|\beta|}:=\int_{1-\varepsilon}^{1}(1-\tau)^{-\frac{1}{2}-\frac{1}{r}} \tau^{-\frac{|\beta|}{2}-1+\frac{n}{2}\left(\frac{1}{q}+\frac{1}{r}\right)} d \tau$.

Analogously, we now estimate $\partial_{x}^{\beta} \nabla v$ :

$$
\begin{aligned}
\left\|\partial_{x}^{\beta} \nabla v(t)\right\|_{q} & \leq\left\|\partial_{x}^{\beta} e^{t \Delta} \nabla v_{0}\right\|_{q}+\left(\int_{0}^{(1-\varepsilon) t}+\int_{(1-\varepsilon) t}^{t}\right)\left\|\partial_{x}^{\beta} \nabla e^{(t-s) \Delta}(v(s)-u(s))\right\|_{q} d s \\
& =: V_{1}+V_{2}+V_{3} .
\end{aligned}
$$

Similarly to the case for $U_{1}$, we estimate $V_{1}$ by

$$
V_{1} \leq \tilde{C}_{4}|\beta|^{\frac{|\beta|}{2}} t^{-\frac{|\beta|}{2}-\frac{n}{2}\left(\frac{1}{n}-\frac{1}{q}\right)}
$$

for $t \in(0, T)$ with the constant $\tilde{C}_{4}:=C_{0}\left\|\nabla v_{0}\right\|_{n}$. For $V_{2}$ we get

$$
\begin{aligned}
V_{2} & \leq \int_{0}^{(1-\varepsilon) t}\left(\left\|\partial_{x}^{\beta} \nabla e^{(t-s) \Delta}\right\|_{\mathcal{L}\left(L^{n} \rightarrow L^{q}\right)}\|u(s)\|_{n}+\left\|\partial_{x}^{\beta} e^{(t-s) \Delta}\right\|_{\mathcal{L}\left(L^{n} \rightarrow L^{q}\right)}\|\nabla v(s)\|_{n}\right) d s \\
& \leq C_{0} \int_{0}^{(1-\varepsilon) t}\left(M_{1}(|\beta|+1)^{\frac{|\beta|+1}{2}}(t-s)^{-\frac{|\beta|+1}{2}-\frac{1}{2}+\frac{n}{2 q}}+M_{3}|\beta|^{\frac{|\beta|}{2}}(t-s)^{-\frac{|\beta|}{2}-\frac{1}{2}+\frac{n}{2 q}}\right) d s \\
& \leq \tilde{C}_{5}(|\beta|+1)^{\frac{|\beta|+1}{2}} \varepsilon^{-\frac{|\beta|}{2}-1+\frac{n}{2 q}} t^{-\frac{|\beta|}{2}+\frac{n}{2 q}}
\end{aligned}
$$

for $t \in(0, T)$ with $\tilde{C}_{5}:=C_{0}\left(M_{1}+M_{3}\right)$, since $\varepsilon<1$ and $t \leq T \leq 1$. We easily estimate 
$V_{3}$ by

$$
\begin{aligned}
V_{3} & \leq \int_{(1-\varepsilon) t}^{t}\left(C_{0}(t-s)^{-\frac{1}{2}}\left\|\partial_{x}^{\beta} u(s)\right\|_{q}+\left\|\partial_{x}^{\beta} \nabla v(s)\right\|_{q}\right) d s \\
& \leq \int_{(1-\varepsilon) t}^{t}(t-s)^{-\frac{1}{2}-\frac{1}{r}} s^{-\frac{1}{2}+\frac{1}{r}}\left(\left\|\partial_{x}^{\beta} u(s)\right\|_{q}+\left\|\partial_{x}^{\beta} \nabla v(s)\right\|_{q}\right) d s
\end{aligned}
$$

since $C_{0} \leq 1$.

Set $\psi(t):=\left\|\partial_{x}^{\beta} u(t)\right\|_{q}+\left\|\partial_{x}^{\beta} \nabla v(t)\right\|_{q}$. Summing up, we see that

$$
\begin{aligned}
\psi(t) \leq & \left(\tilde{C}_{6}|\beta|^{\frac{|\beta|}{2}}+\tilde{C}_{7}(|\beta|+1)^{\frac{|\beta|+1}{2}} \varepsilon^{-\frac{|\beta|}{2}-\frac{3}{2}+\frac{n}{2 q}}+C_{0} \lambda J_{\varepsilon} D_{1}^{2} D_{2}^{|\beta|-2 \delta}|\beta|^{|\beta|-\delta}\right) t^{-\frac{|\beta|}{2}-\frac{1}{2}+\frac{n}{2 q}} \\
& +\left(\tilde{C}_{3}+1\right) \int_{(1-\varepsilon) t}^{t}(t-s)^{-\frac{1}{2}-\frac{1}{r}} s^{-\frac{1}{2}+\frac{1}{r}} \psi(s) d s
\end{aligned}
$$

for $t \in(0, T)$ with some constants $\tilde{C}_{6}:=\tilde{C}_{1}+\tilde{C}_{4}$ and $\tilde{C}_{7}:=\tilde{C}_{2}+\tilde{C}_{5}$ independent of $\beta$ and $t$.

Fix $m_{0} \in \mathbf{N}$ with $m_{0} \geq 2$ large enough so that $J_{m_{0}} \leq 1 /\left(2 \tilde{C}_{3}+2\right)$, where $J_{m}:=$ $J_{1 / m, m}$. Note that $\lim _{m \rightarrow \infty} J_{m} \rightarrow 0$, since $r>2$. Recall that $\varepsilon$ may depend on $|\beta|$, so we take $\varepsilon=1 /|\beta|$ for all $\beta \in \mathbf{N}_{0}^{n}$ with $m=|\beta| \geq m_{0} \geq 2$. In this setting we can easily show that $J_{m}$ is uniformly bounded in $m \geq 2$, indeed, $J_{m} \leq \frac{4 r \sqrt{e}}{r-2}$. Also, for $m \geq 2$ we see $m^{m / 2} \leq m^{m-\delta}$ and

$$
(m+1)^{\frac{m+1}{2}} m^{\frac{m}{2}+\frac{3}{2}-\frac{n}{2 q}} \leq 2(8 m)^{m-\delta} .
$$

We now apply Lemma 2.3 to get

$$
\psi(t) \leq\left(\tilde{C}_{8} 8^{m-\delta}+\tilde{C}_{9} D_{1}^{2} D_{2}^{m-2 \delta}\right) m^{m-\delta} t^{-\frac{m}{2}-\frac{1}{2}+\frac{n}{2 q}}
$$

with $\tilde{C}_{8}:=2 \tilde{C}_{6}+4 \tilde{C}_{7}$ and $\tilde{C}_{9}:=8 C_{0} \lambda r \sqrt{e} /(r-2)$.

Finally, it should be shown

$$
\tilde{C}_{8} 8^{m-\delta}+\tilde{C}_{9} D_{1}^{2} D_{2}^{m-2 \delta} \leq D_{1} D_{2}^{m-\delta}
$$

by suitable choice of the constants $D_{1}$ and $D_{2}$. In fact, let $D_{1} \geq 2 \tilde{C}_{8}$ satisfying (13) for all $\beta \in \mathbf{N}_{0}^{n}$ with $|\beta| \leq m_{0}$. Also, let $D_{2} \geq \max \left(8,\left(2 \bar{C}_{2} D_{1}\right)^{1 / \delta}\right)$, then (14) holds for all $m \geq m_{0}$. Now we obtain (12) for all $\beta \in \mathbf{N}_{0}^{n}$. This completes the proof of Proposition 3.2 .

To end this note we show that

$$
u(t), \nabla v(t) \in C^{\infty}\left(\mathbf{R}^{n}\right), \quad t \in\left(0, T_{0}\right)
$$

by the modification of arguments above. We recall that the mild solution $(u, v)$ is the limit function for successive approximations (6)-(7) by iteration. Take $\beta \in \mathbf{N}_{0}^{n}$ arbitrary. We now define for $j \in \mathbf{N}$

$$
\psi_{j}(t):=\left\|\partial_{x}^{\beta} u_{j}(t)\right\|_{q}+\left\|\partial_{x}^{\beta} \nabla v(t)\right\|_{q},
$$

and argue in the similar way to the proof of Proposition 3.2 to get $\partial_{x}^{\beta} u$ and $\partial_{x}^{\beta} \nabla v \in$ $C\left(0, T_{0} ; L^{q}\right)$ by applying Lemma 2.3 (sequence version). Indeed, there exist constants $D_{1}^{\prime}$ and $D_{2}^{\prime}$ such that

$$
\psi_{j}(t) \leq D_{1}^{\prime}\left(D_{2}^{\prime}|\beta|\right)^{|\beta|-\delta} t^{-\frac{|\beta|}{2}-\frac{n}{2}\left(\frac{1}{p}-\frac{1}{q}\right)}
$$


for all $t \in\left(0, T_{0}\right), \beta \in \mathbf{N}_{0}^{n}$ and $j \in \mathbf{N}$. Since $\beta$ is arbitrary, $u_{j}(t), \nabla v_{j}(t) \in C^{\infty}\left(\mathbf{R}^{n}\right)$ for $t \in\left(0, T_{0}\right)$ and $j \in \mathbf{N}$. Moreover, since $u_{j}$ converges to $u$, and $\nabla v_{j}$ converges to $\nabla v$ in $\left(0, T_{0}\right),(15)$ holds true.

\section{References}

[1] P. Biler, Local and global solvability of some parabolic systems modelling chemotaxis, Adv. Math. Sci. Appl. 8 (1998), 715-743.

[2] R. Denk, M. Hieber and J. Prüss, $\mathcal{R}$-boundedness, Fourier multipliers and problems of elliptic and parabolic type, Mem. Amer. Math. Soc. 166 (2003), no. 788.

[3] H. Fujita and T. Kato, On the Navier-Stokes initial value problem I, Arch. Rational Mech. Anal. 16 (1964), 269-315.

[4] M.-H. Giga and Y. Giga, Nonlinear Partial Differential Equations, Kyouritsu Shuppan, 1999. (in Japanese, English translation to appear).

[5] Y. Giga, K. Inui and S. Matsui, On the Cauchy problem for the Navier-Stokes equations with nondecaying initial data, Quaderni di Matematica 4 (1999), 28-68.

[6] Y. Giga and O. Sawada, On regularizing-decay rate estimates for solutions to the NavierStokes initial value problem, in: Nonlinear Analysis and Applications: to V. Lakshmikantham on his 80th birthday, 1, 2, 2003, 549-562.

[7] D. Henry, Geometric Theory of Semilinear Parabolic Equations, Lecture Notes in Mathematics 840, Springer-Verlag, Berlin-New York, 1981.

[8] C. Kahane, On the spatial analyticity of solutions of the Navier-Stokes equations, Arch. Rational Mech. Anal. 33 (1969), 386-405.

[9] T. Kato, Strong $L^{p}$-solutions of Navier-Stokes equations in $\mathbf{R}^{m}$ with applications to weak solutions, Math. Z. 187 (1984), 471-480.

[10] E. F. Keller and L. A. Segel, Initiation of smile mold agregation viewed as an instability, J. Theor. Biol. 26 (1970), 399-415.

[11] T. Nagai, Blow-up of radially symmetric solutions to a chemotaxis system, Adv. Math. Sci. Appl. 5 (1995), 581-601.

[12] O. Sawada, On analyticity rate estimates of the solutions to the Navier-Stokes equations in Bessel-potential spaces, J. Math. Anal. Appl. 312 (2005), 269-285.

[13] O. Sawada and Y. Taniuchi, On the Boussinesq flow with nondecaying initial data, Funkcial. Ekvac. 47 (2004), 225-250. 
